\title{
A cure for foaming and spill-out in the Melrose oxygenator
}

\author{
R. N. G. ATHERSTONE AND DAVID CHAPMAN \\ From The London Chest Hospital, London, E.2
}

Foaming and spill-out from the drive end of the rotating stainless steel cylinder oxygenator has been a serious problem, but sure remedies for both defects have been discovered. Oxygenation has always been 85 to $100 \%$ even with flow rates over 6 litres/minute. The machine was passed as safe for clinical use after test runs had been made on dogs in 1959, at which time the cause of oxygenator failure was found to be non-filming discs.

At the beginning of 1960 , clinical perfusions were started, and the two problems of spill-out and foaming were encountered.

The minor fault of spill-out is due to incorrect assembly of the first (drive) section of the oxygenator when the next sections are fitted with the large area, oval-holed discs. Blood entering the machine surges to and fro on hitting the plate instead of going through the plate hole. The waves thus formed spill out of the drive end orifice, causing drive failure of the oxygenator. The simple cure is always to assemble the oxygenator with the disc location groove in the first (drive) segment advanced rotationally by one bolt hole from the same groove in the second segment. This advance must be related to the actual rotation direction of the oxygenator.

Foaming, the major fault, which came on within the first 30 minutes of perfusion, was much more difficult to eliminate. Foaming of the blood caused froth to escape out of the drive end orifice in considerable quantities, resulting in loss of blood and failure of the adjacent oxygenator friction drive. More alarming was the collection of much fine froth in the arterial filter, which had to be blown out periodically to prevent the filter becoming completely filled with air bubbles, which would then be forced through the filter screen. Such an air embolism will produce atypical, bizarre, and ill-defined cerebral or other symptoms. There may even be a failure to regain consciousness followed by death.

Details are given of three patients in whom trouble developed during perfusion.
Case 2 was admitted in April 1960 with aortic stenosis. The body surface area was $1.57 \mathrm{~m} .{ }^{2}$ Gross foaming and frothing out developed at $3,500 \mathrm{ml}$./ min., and the modified arterial filter (Atherstone, 1961) had to be blown out repeatedly to prevent it becoming overwhelmed with froth. The oxygenator had to be hand-turned because of drive slip; much blood was lost out of the drive end orifice, and it was very difficult to see the blood level in the oxygenator bell housing because of all the froth.

Unfortunately this patient died on the table due to a failure of the left venting system. There was no discernible evidence of air having got through the filter.

The cause of this apparently unpredictable foaming was investigated, especially as it was found that foaming could not be reproduced by running the machine identically on the residual blood therein after the patient had been disconnected. At that time, detailed examination of the dismantled oxygenator and trombone assemblies yielded no clues.

Case 3 was admitted in May 1960 with pulmonary stenosis. The body surface area was 1.48 $\mathrm{m}^{2}{ }^{2}$ Frothing out, with all its sequelae, recurred at $3,400 \mathrm{ml}$. $/ \mathrm{min}$., and again the modified arterial filter filled with froth. The blood level was difficult to discern, and the oxygenator had to be handturned. Much blood was lost from the machine.

This patient awoke rapidly on the table at the end of the operation and showed no embolic phenomena. He left hospital fit and improved.

Another attempt to reproduce foaming, using the residual blood in the machine, was unsuccessful. Again detailed scrutiny of the oxygenator and trombone assemblies yielded no clue as to the cause of the foaming.

After this case, various changes in the application of M.S. Silicone/Antifoam to parts of the oxygenator and the trombone were made, and foaming vanished completely in the subsequent 29 patients, most of whom were adults requiring flow 
rates well over $3,500 \mathrm{ml} . / \mathrm{min}$. The cure had been effected, but we were then unable to say precisely what the cure was.

Case 32 was admitted in February 1962 with aortic incompetence. The body surface area was $1.82 \mathrm{~m}^{2}$ Some time after starting this $4,300 \mathrm{ml} . /$ min. perfusion, serious frothing out occurred, with all the previously mentioned problems. At one stage during this three-hour perfusion, one of us (R. N. G. A.) was not quick enough in emptying the froth collecting in the arterial filter, and an unknown amount of very fine air bubbles was forced through the overwhelmed filter screen and entered the femoral artery. Although effective measured coronary perfusion had been used, the fact that ventricular defibrillation at $35^{\circ} \mathrm{C}$. took one hour after 55 shocks of 240 volts was very suggestive of air embolism. The patient breathed spontaneously at the end of the operation with satisfactory haemodynamics. However, there was no conscious level and no localizing neurological signs. Death occurred six hours later due to irreversible ventricular fibrillation. Post-mortem examination failed to reveal any evidence of air embolism, which was a very important observation.

Immediately after the operation, detailed examination of the oxygenator, trombone assembly, and the arterial filter gave one clue. Blood filmed readily on the outside areas of the trombone tubes, which had originally been smeared with neat M.S. Silicone/Antifoam. Closer scrutiny showed that this film was missing from these areas. By now it was clear that the root cause of foaming in this oxygenator was an insufficient area of M.S.
Silicone/Antifoam on the trombone tubes. Pre- $\overrightarrow{\vec{D}}$ viously it had been our practice to put the pre- $\bar{\sigma}$ pared trombone assembly in a special linen bag for $\underline{ }$ autoclaving, and on this occasion most of the anti- $\overline{\frac{\bar{N}}{}}$ foam film had been rubbed off on the inside of $\overparen{\nabla}$ the bag during transport and handling.

We therefore designed the cage container illustrated, which ensures that nothing can touch the antifoam film on the trombone tubes, so that the full antifoam barrier is preserved (Figs. 1 and 2). It is essential that the trombone tubes are separately coated overall with a thin film of undiluted M.S. Silicone/Antifoam and then carefully assembled. For accurate and easy assembly, we use a special jig.

Ever since treating case 3 , the oxygenator had always had a thin film of neat M.S. Silicone/Antifoam applied to the inside of the bell reservoir $c$ housing section, its plastic window, and the drive $\stackrel{一}{<}$ end window. Thus it was proved that frothing troubles, with all their dangers, were due to an insufficient antifoam barrier on the trombone assembly.

We are convinced that the safety and efficiency of this and other machines are completely related to the detailed and painstaking care that goes into the preparation thereof, and we are of the opinion that troubles due to foaming are entirely avoid- $\overline{0}$ able. Flows of over 6 litres/minute, with satisfactory oxygenation, have been achieved on a number of occasions.

No pilot will fly an aeroplane without the engineer's check and his own pre-flight, cockpit, and engine run-up checks. Pilot error results in a dead pilot. Pump error results in a damaged or dead

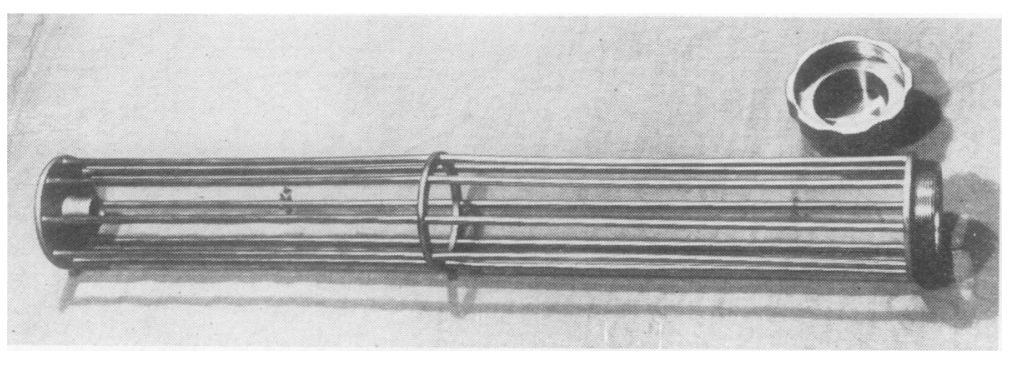

FIG. 1. Carrier empty

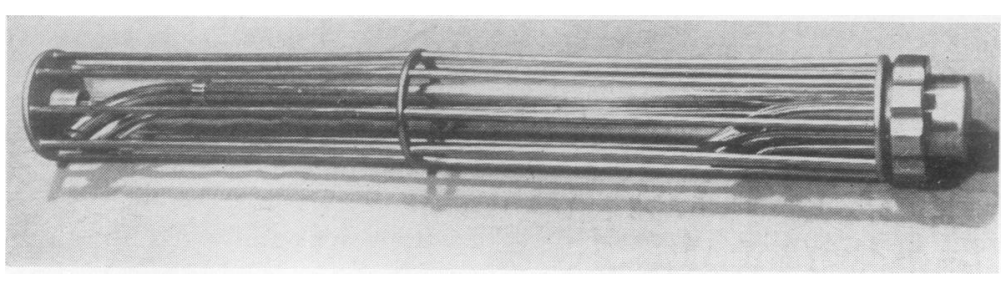

FIG. 2. Trombone in carrier 
patient. The aeroplane is as good as its engineer ; the pump is as good as the technician who has prepared it.

We would like to thank Mr. J. R. Belcher for helpful criticism and advice, and Mr. R. Markham for the photographs.

The trombone carrier was made for us by Endoscopic Instruments Ltd., 62 Shirlands Road, London, W.9.

\section{REFERENCE}

Atherstone, R. N. G. (1961). A modified arterial fitter assembly for the Melrose heart-lung machine. Lancet, 2, 491.

ADDENDUM

Since writing this article, Rheomacrodex has been used in 38 perfusions in adults giving an overall blood dilution of up to $10 \%$. No foaming has been seen. 